\title{
Genetic recombination in a diploid synaptic mutant and a Solanum tuberosum $\times S$. chacoense diploid hybrid
}

\author{
D. S. Douches* and \\ C. F. Quiros
}

Department of Vegetable Crops, University of California, Davis, CA 95616, U.S.A.

A diploid synaptic mutant, M6 with the genotype sy3/sy3,ps/ps, was identified as heterozygous for four enzyme-coding loci, and for the tuber flesh colour locus (Y). Through $4 \times-2 \times$ crosses, half-tetrad analysis was applied to determine the ability of the M6 clone to transmit its heterozygosity to tetraploid offspring. On the average, an 89.03 per cent reduction in recombination was found within the chromosome segments sampled, resulting in 98 per cent transmission of heterozygosity. Cytological observations of microsporogenesis revealed frequent pachytene associations and a high proportion of univalents at diakinesis. These findings show that chromosome pairing in M6 is mostly desynaptic. Diploid and $4 \times-2 \times$ testcrosses were made to study recombination in a $2 n$-pollen producing diploid hybrid T704 $(p s / p s) ; S$. tuberosum L. $\times S$. chacoense Bitt. For the loci compared, recombination was reduced $34 \cdot 56$ per cent on the average. This hybrid transmitted on the average $\mathbf{8 9 . 2}$ per cent of its heterozygosity which was significantly higher than the value previously observed in non-hybrid individuals. Unlike M6, T704 produced both viable $n$ and $2 n$ pollen. In addition, cytological examination of PMCs indicate normal bivalent associations. It is suggested that genomic differentiation between $S$. tuberosum and $S$. chacoense could account for the reduced recombination levels.

\section{INTRODUCTION}

Meiotic abnormalities which lead to the formation of $2 n$ gametes offer unique opportunities to hybridise diploid Solanum species with the cultivated tetraploids for the purposes of increasing genetic diversity and ultimately yield. The benefits of such hybridisations aimed to capture novel genetic diversity are directly related to the meiotic mechanism responsible for diplandroid formation. At present, three meiotic mechanisms have been identified that lead to male $2 n$ gametes in the diploid potato species: parallel and/or fused spindles at metaphase II (First Division Restitution or FDR), premature cytokinesis I, and premature cytokinesis II (Second Division Restitution or SDR) (Mok and Peloquin, 1975; Ramanna, 1979). The discovery of synaptic genes (Iwanaga and Peloquin, 1979) within the diploid potato species has expanded the potential of the FDR mechanisms. The combination of synaptic chromosomes with the parallel spindles gene has opened the possibility of transferring 100 per cent

\footnotetext{
* Present address: Crop and Soil Sciences Department, Michigan State University, East Lansing, MI 48824, U.S.A.
}

of the diploid parent's heterozygosity intact to the tetraploid offspring (Okwuagwa and Peloquin, 1981).

Since Iwanaga and Peloquin (1979) reported the first synaptic mutant (sy1) affecting megasporogenesis in a diploid potato clone, three new synaptic mutants have been identified: sy2 (Johnston et al., 1981); sy3 (Okwaugwu and Peloquin, 1981); and sy4 (Iwanaga, 1984), all affecting microsporogenesis. In these new synaptic mutants, general cytological abnormalities are common. Lack of pairing was noted during diplotene and diakinesis stages, followed by random distribution of the chromosomes during the first division leading to pollen sterility. Okwuagwa and Peloquin (1981) identified the parallel spindle mechanism operating during metaphase II within the sy $3 s y 3$ genotype. This resulted in the fusion of unbalanced nuclei, ultimately leading to balanced and viable dyads.

In asynaptic mutants, homologous pairing fails completely or is incomplete. On the other hand, the desynaptic condition can alter the maintenance of chromosome pairing during pachytene, ultimately reducing fertility as a consequence of 
univalents at diakinesis (Hermsen, 1984a). Definitive cytogenetic characterisation of these synaptic mutants has not been possible. Difficulties lie in the inability to differentiate cytologically between the asynaptic and desynaptic condition. The utilisation of synaptic mutants for the purpose of transferring intact genotypes from parents to offspring rests solely on a theoretical basis, since recombination has been known to occur in synaptic phenotypes of other higher plants (as reviewed by Koduru and Rao, 1981). A study of recombination within chromosome segments of these synaptic mutants should provide experimental data to test the utility of these mutants.

With the production of $2 n$ pollen through the parallel or fused spindle mechanisms, genecentromere mapping is possible through $4 \times-2 \times$ crosses. Mok and Peloquin (1982) proposed the use of electrophoretic variants for mapping while Iwanaga (1984) suggested their application to analyse the genetic consequences of the desynaptic gene sy4. Recently, Douches and Quiros (1987b) determined gene-centromere relationships for 10 enzyme-coding loci. Positioning of electrophoretic markers on the chromosomes enables the identification and characterisation of meiotic mutants by genetic analysis in the potato.

In this paper, M6, a diploid synaptic mutant with the ability to generate FDR $2 n$ pollen through the parallel spindles mechanism (sy3/sys3,ps/ps), was analysed through $4 \times-2 \times$ crosses and subsequent half-tetrad rationale, in conjunction with cytological observations of microsporogenesis. The resulting recombinational frequencies were compared with recombinational values obtained in non-mutant plants to quantify the reduction in recombination. The same approach was applied to determine the level of recombination in an interspecific hybrid of $S$. tuberosum L. and $S$. chacoense Bitt. as a means to detect genomic differentiation between these two species. This work provides experimental data on the extent of transmission of heterozygosity for synaptic mutants and interspecific hybrids to their progenies.

\section{MATERIAL AND METHODS}

\section{Plant material}

The diploid synaptic, parallel spindles, double mutant, M6 (sy3/sy3,ps/ps) $(2 n=2 \times=24)$, and the interspecific hybrid, T704 ( $p s, p s)(2 n=2 \times=$ 24), were kindly supplied by Dr S. J. Peloquin
(University of Wisconsin, Madison). M6 was selected from the progeny of the cross W5295.7 (I) $\times$ W5337.3 $(J)$, both of which were species hybrids between $S$. tuberosum dihaploids $(2 n \times 2 \times=24)$ and $S$. phureja Juz. et Buk. $(2 n=2 \times=24)$. T704 is a diploid species hybrid between a $S$. tuberosum dihaploid and $S$. chacoense. These diploids were crossed to tetraploid clones. The tetraploid parents used in the $4 \times-2 \times$ and $2 \times-4 \times$ crosses were Lemhi Russet, NDD277-2, BC8370-4, T245.7, and PAS3006-7. These clones were kindly supplied by Dr R. E. Voss (University of California, Davis). Diploid crosses were made in the direction $84 S 10 \times$ T704 and T704 $\times 85$ SD56. 84 S10 and 85SD56 are diploid clones of $S$. phureja origin.

\section{Electrophoresis}

Employing horizontal starch gel electrophoresis, eight enzyme systems were studied which revealed nine enzyme-coding loci: Dia-1 (diaphorase), Got1 (glutamate oxaloacetate transminase), Idh-1 (isocitrate dehydrogenase), $M d h-1$ (malate dehydrogenase), 6-Pgdh-3 (6-phosphogluconate dehydrogenase), Pgm-2 (phosphoglucomutase), Prx-2, Prx-3 (peroxidase), and $S d h-1$ (shikimic acid dehydrogenase). Inheritance data and allozyme patterns for these loci are described by Quiros and McHale (1985) and Douches and Quiros (1987a). Excluding Dia-1 and Prx-2, gene-centromere map distances were previously estimated (Douches and Quiros, 1987b). Specific electrophoretic procedures are described by Quiros (1981). Enzyme stains used are according to Vallejos (1983).

To test for recombination, $4 \times-2 \times($ FDR $)$ testcrosses were made for the enzyme loci listed above and $Y$ determining tuber flesh colour (Howard, 1970). Since a single $4 \times$ parent was not identified to be nulliplex for all the loci tested, a series of crosses were made using Lemhi Russet, NDD2772, BC8370-4, Y245.7, and PAS3006-7. The genotypes of these clones are shown in tables 1 and 2. Gene-centromere map distances for these loci including Dia-1 and Prx-2 were previously estimated by Douches and Quiros (1987b) using diploid stocks from the $S$. tuberosum group. These values were used as standards to compare recombinational frequencies in M6 and T704. Genecentromere map distances are simply another way of expressing the recombination frequency for a chromosome segment between a locus and its centromere.

Under normal synaptic conditions, a $4 \times-$ $2 \times($ FDR $)$ testcross would generate progeny that 
can be divided into three classes (assuming diallelic loci): simplex, nulliplex, and duplex genotypes, with the second and third classes indicating a recombinational event. In case of dominance, only the nulliplex progeny would be diagnostic; however, with these co-dominant electrophoretic markers, the duplex genotype can be distinguished from the simplex on the basis of asymmetric banding intensities (Martinez-Zapater and Olivier, 1985). Under asynaptic conditions assuming zero recombination, these $4 \times-2 \times$ testcrosses should yield exclusively simplex genotypes. If crossing-over does occur, nulliplex and duplex progeny should be recovered. The greater the distance of a gene from its centromere, the better the opportunity for detecting crossingover in M6 and T704.

All crosses were made in the greenhouse. Offtypes observed in $4 \times-2 \times$ offspring were subjected to chromosome counts in either root-tip or another squashes to rule out triploids. No triploids were observed, presumably due to a strong triploid block (Marks, 1966; Hanneman and Peloquin, 1968; Hermsen, 1984b). Germinated seedlings were transplanted into trays ( 50 plants per tray). In 4 to 6 weeks, root or leaf samples from healthy and vigorously growing seedlings were sampled for electrophoretic analysis. To determine tuber flesh colour ( $Y$ locus), the seedlings were allowed to tuberise in the trays. Small mature tubers were harvested in three to four months. Tubers were then visually compared with parental tubers for flesh colour determination.

To cytologically study diplandroid formation, flower buds were collected and immediately fixed in a freshly prepared ethanol/propionic acid solution $(3: 1)$ with ferric chloride added as a mordant (Swaminathan et al., 1954). After 24 hours, the buds were removed from the fixative, rinsed, and stored in 70 per cent ethanol at $4^{\circ} \mathrm{C}$. Anthers were dissected and squashed in 1 per cent acetocarmine. All meiotic stages of the pollen mother cells were examined. The occurrence of parallel and/or fused spindles during Metaphase II, followed by dyad formation identified the FDR mode of $2 n$ pollen formation (Mok and Peloquin, 1975; Ramanna, 1979). Pollen staining was in accordance with Quinn et al. (1974).

Table 1 Half-tetrad analysis of electrophoretic markers in $4 \times-2 \times$ crosses with $M 6$, a synaptic/parallel spindles mutant

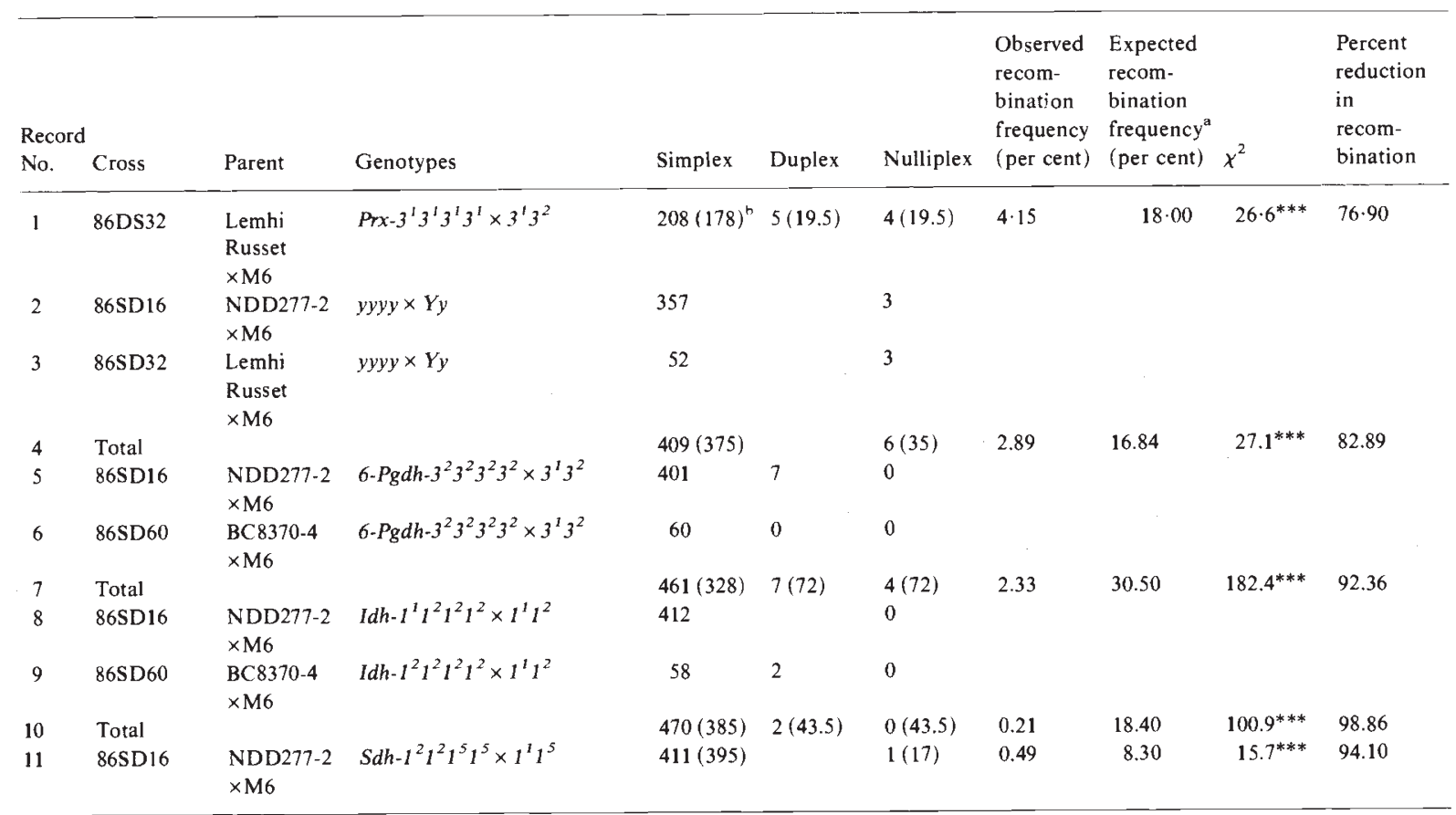

${ }^{a}$ From Douches and Quiros, $1986 b$

${ }^{b}$ Expected values in parentheses 
Table 2 Half-tetrad analysis of electrophoretic markers in $4 \times-2 \times$ testcrosses with $T 704$, an interspecific hybrid of $S$. tuberosum $\times S$. chacoense

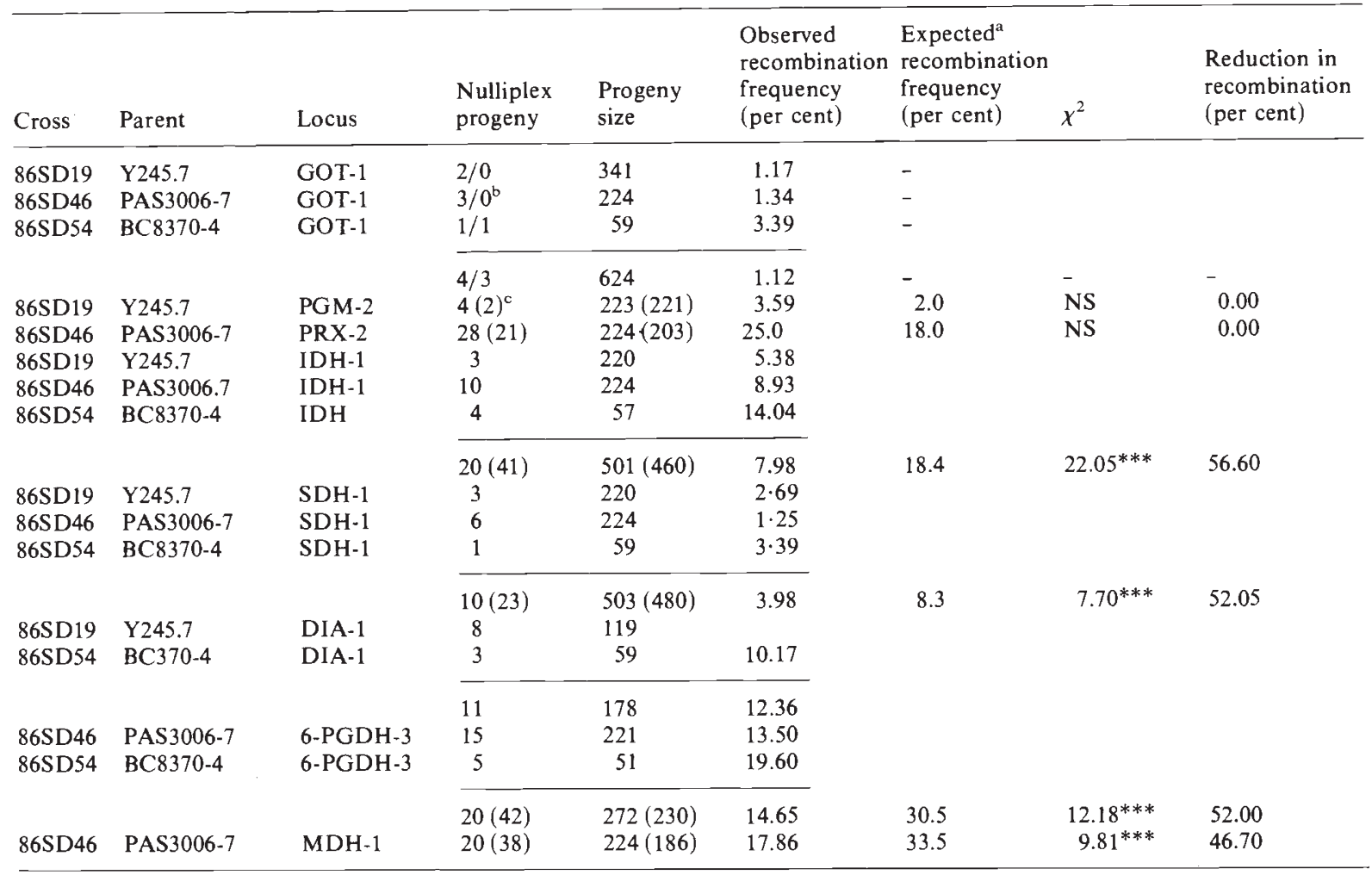

${ }^{a}$ From Douches, D. S. and C. F. Quiros, $1987 b$

${ }^{b}$ Nulliplex/duplex genotypes

${ }^{c}$ Expected value in parentheses

${ }^{d}$ Expected value for Prx-3-centromere linkage block

*** Probability $>0.001$

\section{RESULTS}

\section{Extent of recombination in the synaptic mutant $M 6$}

Electrophoretic analysis of M6 revealed that it was heterozygous for four isozyme loci: $I d h-1^{1} 1^{2}, S d h$ $1^{1} 1^{5}, \operatorname{Pr} x-3^{1} 3^{3}$ and $6-\operatorname{Pgdh}-3^{1} 3^{2}$. In addition, M6 was heterozygous for yellow tuber flesh as determined by the genotype of its parents (Masson, 1985). Five chromosome segments of varying lengths were therefore available to study and test for the occurrence of recombination in the synaptic mutant M6 (table 1).

The 6-Pgdh-3 locus was calculated to be $30 \cdot 1 \mathrm{~m}$.u. from its centromere in crosses between Group Tuberosum accessions (Douches and Quiros, 1987b). Thus, this locus provided the best opportunity to detect cross-over in M6 because of its distal chromosome arm position. A recombination value of 2.33 per cent was obtained for M6 after pooling segregation data from two tetraploid progenies, 86SD16 and 86SD60 $\left(\chi^{2}=3.071, P=\right.$ 0.07 ) (table 1).

Segregation for the Idh-1 locus, located at 18.4 m.u. from the centromere, was also amenable to half-tetrad analysis in the M6 progenies. In the family 86DS60, two recombinant duplex individuals were observed from 60 plants. However, in the family, $86 \mathrm{SD} 16$, the analysis was not straightforward. The tetraploid parent, NDD277-2, as determined by progeny testing, was a simplex heterozygote $\left(\operatorname{Idh}-1^{1} 1^{2} 1^{2} 1^{2}\right)$ rather than homozygous for the locus as required by halftetrad analysis. As a result, only half of the progeny was amenable to the half-tetrad rationale. Recombination was not detected in this cross as nulliplex genotypes were not found. 
Segregation of the $S d h-1$ locus, located $8 \cdot 3$ m.u. from the centromere, was studied in the family 86DS16. Since the tetraploid parent was a duplex heterozygote $\left(S d h-1^{2} 1^{2} 1^{5} 1^{5}\right)$ and M6 was heterozygous with the alleles $S d h-1^{1} 1^{5}$, only one of the recombinant progeny classes was observable in the cross. A 0.49 per cent recombination rate was detected for this gene-centromere segment in a progeny of 412 plants.

The cross between Lemhi Russet and M6 (86SD32) estimated a $4 \cdot 15$ per cent recombination rate in the chromosome region between the Prx-3 locus and its centromere. The expected rate for this locus was 18.0 per cent.

Recombination between the $Y$ locus and its centromere was evaluated in the same family, 86SDS32, along with 86SD16. Based upon the frequency of white-fleshed tubers (table 1), pooled families $\left(\chi^{2}=3.83, P=0.06\right)$ revealed a 2.89 per cent rate for this proximal arm region compared with the normal location of 16.8 m.u. from the centromere.

\section{Cytological analysis of microsporogenesis in M6(sy3/sy3,ps/ps)}

General asynchrony was found in the meiotic process. At times, single anther squashes revealed meiotic stages from prophase I to dyad formation. During pachytene, meiocytes were identified with varying degrees of chromosome pairing. Complete synapsis was identified frequently, suggesting that some level of recombination may be occurring. (fig. 1A). Diakinesis was generally characterised by cells showing mainly univalents, hence revealing the desynaptic nature of this clone. Bivalent formation was very low with a range of zero to three bivalents per cell (fig. 1B).

The chromsomes of M6 during metaphase I lacked normal orientation on the equatorial plate, hence anaphase I was not clearly distinguishable from metaphase I. Spindles were elongated with a curved orientation (fig. 1C). Lagging chromosomes were regularly observed during the first division, leading to abnormal chromosome distri-
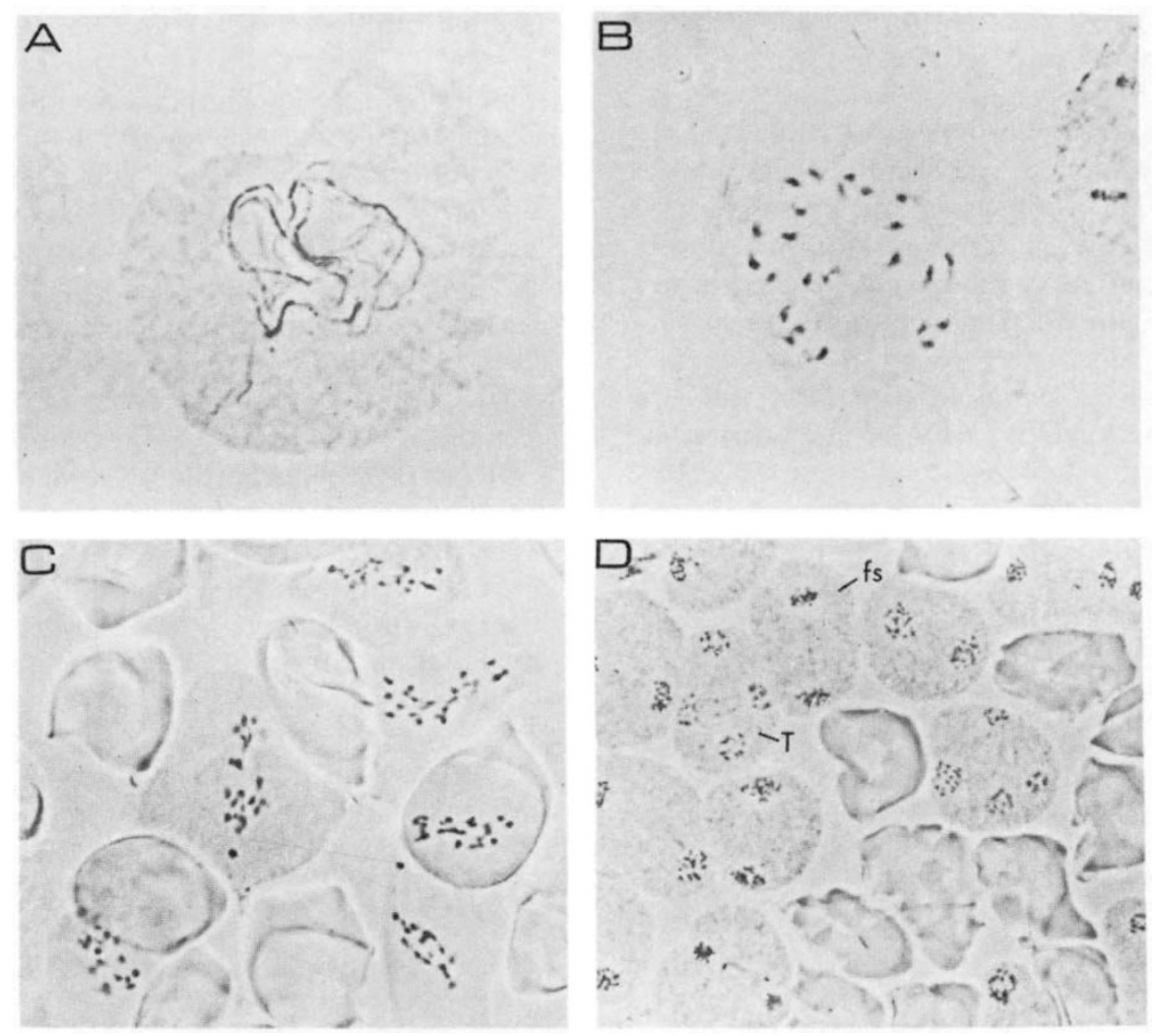

Figure 1 Various stages of meiosis in pollen mother cells of the desynaptic, diploid clone M6. (A) Pachytene showing mostly bivalents. (B) Diakinesis with cell showing mainly univalents. (C) Metaphase/anaphase I characterised by unbalanced chromosome segregation and curved, elongated spindles. (D) Telophase II with fused spindle orientation (fs). Note tetrad formation. 
bution. Bivalent frequencies within these meiocytes ranged from zero to four; however, most cells lacked clear bivalent associations. Chromosome imbalance was noted at the end of the first division. At times bivalent separation was observed during late anaphase (fig. 1C). Precocious chromatid separation of univalents was not evident. Metaphase II was characterised by a strong expression of the parallel/fused spindles mechanism. Anaphase II and telophase II were typical of psps. Expression of tripolar spindles and normal spindle orientation with balanced tetrads was evident at low frequencies (fig. 1D). An estimate of pollen fertility, based upon the frequency of plump, stainable pollen, was 36.0 per cent.

\section{Extent of recombination in the interspecific hybrid T704}

Electrophoretic analysis of T704, a S. tuberosum $\times$ $S$. chacoense species hybrid, revealed eight heterozygous enzyme-coding loci: Dia-1, Got-1, Idh-1, Mdh-1, 6-Pgdh-3, Pgm-2, Prx-2 and Sdh-1. Unlike M6, T704 yielded progenies in both $4 \times-2 \times$ and $2 \times-2 \times$ crosses because of viable $2 n$ and $n$ pollen. Recombination frequencies were studied in progenies of both ploidy levels.

The $4 \times-2 \times$ rationale applied to the synaptic mutant was similarly extended to the $4 \times-2 \times$ crosses involving T704 as the pollen parent. A series of three $4 \times-2 \times$ crosses and two $2 \times-2 \times$ crosses were made to estimate the recombination rates for the eight different loci and two linkage blocks (table 2).

The standard recombination rate for the chromosomal segment between $M d h-1$ and its centromere was estimated to be 33.5 per cent. In a $4 \times-2 \times$ cross involving T704, a rate of 17.86 per cent was estimated from a progeny of 224 plants in family 86DS46 (table 2).

For $6-P g d h-3$, located at $30 \cdot 1$ m.u. from its centromere, a recombination value of 14.65 per cent was obtained for T704 after pooling the segregating data from progenies $86 \mathrm{SD} 46$ and $86 \operatorname{SD} 54\left(\chi^{2}=\right.$ $0.48, P=0.53$ ).

A combined estimate of 7.98 per cent was found for T704 after pooling the three families for segregation of the Idh-1 locus $\left(\chi^{2}=2 \cdot 514, P=0 \cdot 30\right)$. This rate was significantly reduced from the expected rate of 18.4 per cent. The $S d h$-1-centromere recombination value in the same three families $\left(\chi^{2}=0.987, P=0.63\right.$ ) was reduced to 3.90 per cent from the standard rate of $8 \cdot 3$ per cent.

No reduction in recombination was found for the chromosome region between $\mathrm{Pgm}-2$ and its centromere. Based upon a fit to chi-square expectations, the present estimate of 3.58 per cent was not significantly different from the expected value of $2 \cdot 00$ per cent.

The highest recombination rate ( 25 per cent) for any chromosome segments studied in T704 was found within the $\operatorname{Pr} x$-2-centromere segment in family 86 SDS46 of 224 plants.

Segregation of the Dia-1 and Got-1 loci were also studied in these $4 \times-2 \times$ testcrosses: however, no standard estimates of the recombination frequencies exist to compare these values. The pooled families 86SD19 and 86SD54 $\left(\chi^{2}=0 \cdot 182\right.$, $P=0.70$ ) estimated a 12.36 per cent rate for the Dia-1-centromere segment in T704. Segregation of the Got-1 locus was studied in 86SD19, 86SD46, and $86 \mathrm{SD} 54$. A pooled estimate of all three families $\left(\chi^{2}=2 \cdot 82, P=0 \cdot 12\right)$ places Got 1 proximal to its centromere with a $1 \cdot 12$ per cent rate.

The reciprocal $2 \times-4 \times$ cross involving T704 was unsuccessful because of little or no expression of $2 n$ egg formation, however, T704 was used as the pistillate parent in a diploid cross (86SD47). A 17 per cent recombination rate was observed for the $I d h-1 / S d h-1$ linkage block, which was reduced from the standard value of $36 \cdot 8$ per cent (table 3 ). An earlier cross between 84S10 and T704 (86SD10) estimated a 10.4 per cent recombination frequency for this same linkage block, while a $32 \cdot 4$ per cent rate was obtained between 6-Pgdh-3 and Dia-1 (Douches and Quiros, 1987a).

Table 3 Diploid testcross data with T704, an interspecific hybrid of $S$. tubersum $\times S$. chacoense

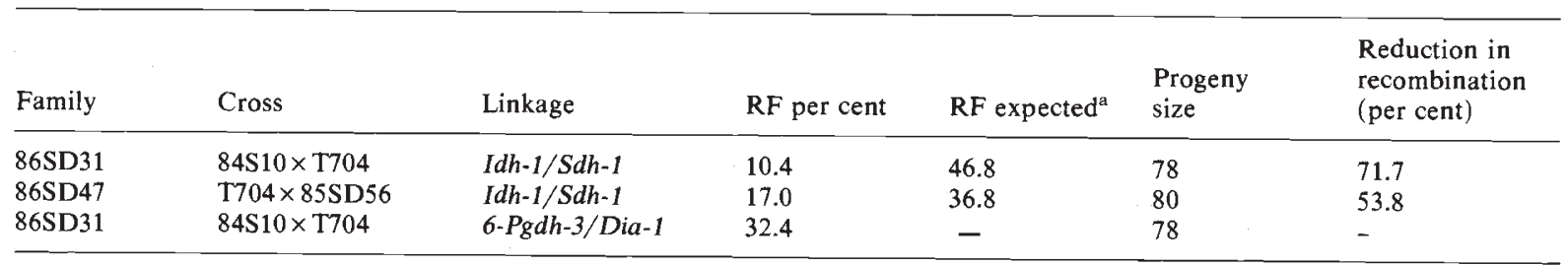

\footnotetext{
${ }^{a}$ From Douches and Quiros (1986a)
} 


\section{Cytological observations of T704}

Examination of PMCs confirmed a strong expression of the parallel/fused spindles mechanism leading to $2 n$ pollen formation. Analysis of early stages found normal chromosome pairing during pachytene and bivalent formation in diakinesis. Normal chromosome disjunction followed. Pollen fertility was high (>90 per cent).

\section{DISCUSSION}

\section{Genetic and cytological analyses of M6}

In $4 \times-2 \times$ testcrosses with M6, five opportunities were available to sample this clone's ability to transmit its heterozygosity. Despite general pachytene pairing, all five chromosome segments of M6 showed severe reductions in crossing-over throughout the genome. The average reduction in crossing-over from expected was 89.03 per cent with a range of $76.94-98.86$ per cent. Under normal synaptic conditions, these five regions would have transferred an average of 81.59 per cent of their heterozygosity. Considering the reduction in recombination under the abnormal synaptic conditions conferred by the sy3 gene, 97.99 per cent of M6's heterozygosity was transmitted to its progeny.

The genotypes combining the sy3 gene and the ps genes were first described by Okwuagwu and Peloquin (1981), however, pachytene stages were not mentioned. In many respects, our cytological observations in M6 agree with those of Okwuagwu and Peloquin. The $s y 1$, sy2, and $s y 3$ phenotypes have similar expression, but $s y 1$ was expressed only in megasporogenesis. Ramanna (1983) observed from $1 \cdot 1$ to $8 \cdot 3$ II per cell during metaphase $I$ in desynaptic plants derived from the parents $(I \times J)$ of M6. This range of restricted pairing could suggest a variable expression for the desynaptic genes as influenced by other genotypic factors and interactions.

The cytological similarities between sy4 and sy3 were striking. Iwanaga's (1984) cytological description of the synaptic abnormalities matched closely with the present description of the sy3 in M6. He noted a consistent low frequency of synapsis during pachytene and diakinesis. Thus, Iwanaga predicted a 97 per cent transmission of heterozygosity for this mutant, based upon a frequency of 3 chiasmata per meiocyte.

Hermsen and Ramanna (1983) suggested that desynaptic genes would be expressed in both micro- and megasporogenesis. No fruit set was obtainable in $2 \times-4 \times$ crosses with M6. Lack of seed set may be attributed to either lack of expression of the sy3 gene in the female side or lack of a restitution mechanism. Cytological examination of megasporogenesis would be needed to discriminate between these two possibilities.

Although the cytogenetic data indicated that M6 is desynaptic; i.e. generalised pairing during pachytene and high frequency of univalents at diakinesis, the $4 \times-2 \times$ genetic data revealed a reduced recombination typical of an asynaptic mutant. This indicated that the sy3 mutation interferes with chiasma formation leading to a premature separation of the bivalents in diakinesis. The theoretical expectation of 100 per cent transfer of the parent's heterozygosity to the offspring was close to being realised in M6. Thus, this restricted recombination caused the transfer of a significantly greater ( 81.54 per cent vs. 97.99 per cent) amount of heterozygosity than the ps gene alone. The potential value of the $s y 3 / s y 3$, ps/ps genotype for $4 \times-2 \times$ breeding schemes to develop highly uniform, heterozygous progenies for true seed production (TPS), is demonstrated. However, the utility of the sy 3 gene in the development of an apomictic system would be questionable because of its potential for recombination.

\section{Genetic analysis of $T 704$}

In comparison to M6, crossing-over occurs much more frequently in the interspecific hybrid T704. Of the loci sampled, an average recombinational frequency of 2.01 per cent was found in M6, while T704 had 10.82 per cent, a five-fold increase, whereas recombination in normal FDR clones had a 18.55 per cent recombination frequency. In $4 \times-$ $2 \times$ testcrosses, the recombination rate for six chromosome regions were compared to expected values for their respective regions. Reduction in crossing-over in T704 ranged from 0 to 56.60 per cent, with an average of 34.56 per cent. Considering the eight gene-centromere segments sampled in these $4 \times-2 \times$ testcrosses, the average heterozygosity transferred was $89 \cdot 18$ per cent. Based upon $\chi^{2}$ comparisons of observed and expected recombination rates for individual loci, a significantly greater amount of heterozygosity is transferred to the tetraploid offspring (89.18 per cent vs. 81.45 per cent respectively) using T704.

For the four chromosome arm regions where reduction in recombination was significant, the decrease regressed linearly. For each recombination value observed, the reduction averaged 51.84 per cent regardless of the expected recombinational frequency for the respective segments. 
The chromosome arm region involving $\mathrm{Pgm}-2$ did not deviate from the expected value. Based upon chi-square tests, a 3.59 per cent recombination frequency for the segregation of the Pgm-2 locus was not significantly different from the expected value of 2.0 per cent. It can be argued that a significant reduction in crossing-over would be difficult to detect statistically in small chromosome segments with population sizes of approximately 200 .

A pooled recombination frequency of $1 \cdot 12$ per cent was observed for the segregation of the Got-1 locus in $4 \times-2 \times$ testcrosses. No additional $4 \times-2 \times$ crosses have been available using different diploid parents for a comparison. Applying the reasoning set forth for the Pgm-2 segregation along with $2 \times-4 \times$ (unpublished) and $4 \times-4 \times$ data (Douches and Quiros, 1987b), it is likely that the Got-1 recombination frequency obtained expresses a true proximal position for this locus.

The highest recombination rate $(25$ per cent $)$ for any chromosome segments studied in T704 was found within the Prx-2-centromere segment. At this time no direct estimates for this segment exist, but it is known that $\operatorname{Pr} x-2$ and $\operatorname{Pr} x-3$ are tightly linked with 0.6 map units separating the two loci (Douches and Quiros, $1987 a$; Quiros and McHale, 1985). Hence, the Prx-3-centromere linkage (18.0 m.u.) was substituted as the reference. Knowing this relationship, the observed Prx-2centromere recombination rate was deduced to be not significantly different than expected. With this linkage block of 18.0 m.u., a reduction in recombination would have been expected. In comparison, Idh-1 segregation has a similar rate of 18.4 per cent under normal instances. Within T704, a rate for this locus was reduced to 7.98 per cent. The differential rates for the two loci situated at equivalent distances from their centromeres indicate different degrees of chromosome homology between the two genomes. This conclusion is supported by higher recombination frequencies found in certain chromosome segments of higher exhibiting de- or asynaptic conditions (Rick, 1971; Koduru and Rao, 1981).

In the diploid cross where T704 was used as the pistillate parent, a 53.80 per cent reduction in expected recombination was seen for the $I d h$ $1 / S d h-1$ linkage block. The reduction in crossingover regressed in a similar fashion as the chromosome segments studied in microsporogenesis. Previously half-tetrad analysis and diploid testcross data using standard diploid stocks positioned the $S d h-1$ and $I d h-1$ on opposite chromosome arms (Douches and Quiros, 1987b). The recombination rate for this linkage block in T704 agrees with the $4 \times-2 \times$ data for the $I d h-1$ and $S d h-1$ genecentromere segments when T704 was also used as the pollen parent.

Since Dia-1 and 6-Pgdh-3 are found on the same chromosome it can be assumed that the recombination frequency within the Dia-1centromere segment was reduced at a similar rate as 6 -Pgdh-3. Hence, we can estimate a normal recombination frequency of 25.73 per cent for the Dia-1-centromere segment. If the predicted recombination value for the Dia-1-centromere segment and the expected value for the 6-Pgdh-3centromere are summed, a $56 \cdot 23$ m.u. map distance is then predicted. Under normal synaptic conditions, this linkage block had not been detected. It is possible that in utilising T704, we were able to take advantage of the reduced recombination levels to identify this new linkage block and position the loci in relation to their centromere. The usefulness of this interspecific hybrid in assigning new markers to linkage groups could continue to be realised until more markers saturate the genome.

Douches and Quiros (1987b) reported genecentromere map distances for ten enzyme-coding loci and the $Y$ locus. Their investigation and the current study suggest that these markers are distributed over 11 chromosome arms of the potato genome. With the current $4 \times-2 \times$ linkage data for Dia-1 and 6-Pgdh-3, we assign Dia-1 and 6-Pgdh-3 to opposite chromosome arms. The genomic distribution of these 12 loci is now expanded to 12 chromosome arms.

\section{Genomic differentiation in T704}

Unlike M6, the reduction in recombination in 7704 must arise from a different mechanism. Besides the formation of parallel spindles, other meiotic or chromosome abnormalities were not noted in T704. Reduction in crossing over was not expected in this clone since both parental species ( $S$. tuberosum and $S$. chacoense) are supposed to have the same genome (Hawkes, 1978).

Our findings, however, raise the question of genomic differentiation between $S$. tuberosum and $S$. chacoense. This is supported by distorted segregations for several genes in $S$. tuberosum $\times S$. chacoense progenies (Quiros and McHale, 1985; Douches and Quiros, 1987a). Hawkes (1978) assigns the genomic formula $A 1$ to these two species, which belong to the South American subgroup. Most of the diploid species of this subgroup hybridise readily and produce fertile $F_{1}$ hybrids. 
From pairing studies, Hawkes concluded that genomic differentiation in the conventional sense has not progressed very far among the South American diploid species. Ramanna and Hermsen (1979) suggest differentiation has not resulted in pairing failure at meiosis. However, when Swaminathan (1953) doubled the chromosomes of $S$. rybinii $\times S$. tuberosum and $S$. chacoense $\times S$. tuberosum species hybrids, quadrivalent frequencies were reduced in the $S$. $c h c \times t u b$ hybrids, suggesting low levels of genomic differentiation. To account for observations like these, Dvorak (1983) suggested that the genotypes of the diploid species act in a way to suppress heterogenetic pairing. The segregation data for T704 reported in this paper may supply evidence to support this proposal of genomic differentiation. Further work must be done with other accessions of $S$. chacoense and other diploid species to ascertain the significance of this question.

\section{REFERENCES}

DOUCHES, D. S. AND QUIRoS, C. F. $1987 a$. Additional isozyme loci in tuber-bearing Solanums: Inheritance and linkage relationships. $J$. Heredity (in press).

DOUCHES, D.S. AND QUIROS, C. F. 1987 b. Use of $4 \times-2 \times$ crosses to determine gene-centromere map distances of isozyme loci in Solanum species. Can. J. Genet. Cytol. (in press).

DVORAK, J. 1983. Evidence for genetic suppression of heterogenetic chromosome pairing in polyploid species of Solanum sect Petota. Can. J. Genet. Cytol., 25, 530-539.

HANNEMAN, R. E. AND PELOQUIN, S. J. 1968. Ploidy levels of progeny from diploid-tetraploid crosses in potato. Am. Potato J., 45, 255-61.

haWkes, J. G. 1978. Biosystematics of the Potato. Harris, P. M. (ed.) In The Potato Crop: The scientific basis of improvement, Chapman and Hall, London, pp. 15-67.

HERMSEN, J. G. TH. 1984a. Mechanisms and genetic implications of $2 n$-gamete formation. Iowa State J. Res., 58, 421434.

HERMSEN, J. G. TH. 1984 b. The potential of meiotic polyploidization in breeding allogamous crops. Iowa State Je. Res., $58,435-448$.

HOWARD, H. W. 1970. Genetics of the potato. Springer-Verlag, New York.

IWANAGA, M. 1984. Discovery of a synaptic mutant in potato haploids and its usefulness for potato breeding. Theor. Appl. Genet., 68, 87-93.

IWANAGA, M. PELOQUIN, S. J. 1979. Synaptic mutant affecting only megasporogenesis in potatoes. J. Hered., 70, 385-389.
JOHNSTON, S. A., HANNEMAN, R. E. JR, AND RUHDE, R. W. 1981. Identification of an asynaptic variant in Solanum commersonii. Am. Potato J., 58, 506.

JONGEDIJK, E. 1983. Selection for first division restitution $2 n$-egg formation in diploid potatoes. Potato Res., 26, 399.

KODURU, P. R. K. AND RAO, M. K. 1981. Cytogenetics of synaptic mutants in higher plants. Theor. Appl. Genet., 59, 197214.

MARKS, G. E. 1966. The enigma of triploid potatoes. Euphytica, $15,285-290$.

MARTINEZ-APATER, J. M. AND Olivier, J. L. 1984. Genetic analysis of isozyme loci in tetraploid potatoes (Solanum tuberosum L.) Genetics, 108, 669-679.

MASSON, M. 1985. Mapping, Combining abilities, heritabilities and heterosis with $4 \times-2 \times$ crosses in potato. PH.D. Thesis, University of Wisconsin, Madison.

MOK, D. W. S. AND PELOQUIN. S. J. 1975. Three mechanisms of $2 n$ pollen formation in diploid potatoes. Can. J. Genet. Cytol., 17, 217-225.

MOK, I. G. AND PELOQUIN, S. J. 1982. Sexual polyploidization and protein diversity in potatoes. Am. Potato J., 59, 480.

OKWUAGWU, C. O. AND PELOQuiN, S. J. 1981. A method of transferring the intact parental genotype to the offspring via meiotic mutants. Am. Potato J., 58, 512-513.

QUINN, A. A., MOK, D. W.S. AND PELOQUIN, S. J. 1974. Distribution and significance of diplandroids among the diploid Solanums. Am. Potato J., 51, 16-21.

QUIROS, C. F. 1981. Starch gel electrophoresis techniques used with alfalfa and other Medicago species. Can. J. Plant. Sci., $61,745-749$.

QUIROS, C. F. AND MCHALE, N. 1985. Genetic analysis of isozyme variants in diplooid and tetraploid potatoes. Genetics, 111, 131-145.

RAMANNA, M. S. 1979. A re-examination of the mechanisms of $2 n$ gamete formation in potato and its implications for breeding. Euphytica, 28, 537-561.

RAMANNA, M. S. 1983. First division restitution gametes through fertile desynaptic mutants of potato. Euphytica, $32,337-350$.

RAMANNA, M. S. AND HERMSEN, J. G. Th. 1979. Genome Relationships in tuber-bearing Solanums Hawkes, J. G., Lester, R. N. and Skelding, A. D. (eds.) In The biology and taxonomy of the Solanceae Academic Press, New York, pp. 647-657.

RICK, C. M. 1971. Some cytogenetic features of the genome in diploid plant species. Stadler Symposia, Vol. 1 and 2, 153 174.

SWAMINATHAN, M. S. 1953. Studies on the inter-relationships between taxonomic series in the section Tuberarium, Genus Solanum. I. Commersoniana and Tuberosa Am. Potato J. 30, 271-281.

SWAMINATHAN, M. S., MAGOON, M. L. AND MEHRA, K. L. 1954. A simple propionic-carmine PMC smear method for plants with small chromosomes. Indian J. Genet. Plant Breed., 14, 87-88.

VALLEJOS, C. E. 1983. Enzyme activity staining. (eds.) Tanksley, S. D. and Orton, T. J. In Isozymes in plant genetics and breeding Vol A. Elsevier, Amsterdam, pp. 469-516. 